\title{
ON BOUNDARY BEHAVIOUR OF SYMPLECTOMORPHISMS
}

\author{
Elisabetta Barletta ANd Sorin Dragomir
}

Let $\Omega \subset C^{n}$ be a strictly pseudoconvex domain, $\gamma$ an admissible weight, and $K_{\gamma}(z, \zeta)$ the reproducing (or $\gamma$-Bergman) kernel for $L^{2} H(\Omega, \gamma)$, the space of square integrable functions, with respect to the measure $\gamma d \mu$, which are holomorphic in $\Omega\left(d \mu\right.$ is the Lebesgue measure in $\left.\boldsymbol{R}^{2 n}\right)$, cf. e.g. Z. Pasternak-Winiarski [17]. Consider the complex tensor field:

$$
H_{\gamma}=\sum_{1 \leq l, J \leq n}\left(\frac{\partial^{2}}{\partial z_{i} \partial \bar{z}_{J}} \log K_{\gamma}(z, z)\right) d z_{l} \otimes d \bar{z}_{J}
$$

and the corresponding real tangent $(0,2)$-tensor field $g_{\gamma}$ given by:

$$
g_{\gamma}=\operatorname{Re}\left\{\left.H_{\gamma}\right|_{\chi(\Omega) \times \chi(\Omega)}\right\},
$$

where $\chi(\Omega)$ is the $C^{\infty}(\Omega)$-module of all real tangent vector fields on $\Omega$. Under suitable conditions (cf. section 2) $g_{\gamma}$ is a Kählerian metric on $\Omega$, hence $\omega_{\gamma}=$ $-i \partial \bar{\partial} \log K_{\gamma}(z, z)$ is a symplectic structure (the Kähler 2-form of $g_{\gamma}$ ). One of the problems we take up in the present paper may be stated as follows. Let $F$ : $\Omega \rightarrow \Omega$ be a symplectomorphism of $\left(\Omega, \omega_{\gamma}\right)$ in itself, smooth up to the boundary. Does $F: \partial \Omega \rightarrow \partial \Omega$ preserve the contact structure of the boundary?

Our interest may be motivated as follows. If $F: \Omega \rightarrow \Omega$ is a biholomorphism then, by a celebrated result of C. Fefferman (cf. Theorem 1 in [4], p. 2) $F$ is smooth up to the boundary, hence $F: \partial \Omega \rightarrow \partial \Omega$ is a $\mathrm{CR}$ diffeomorphism, and in particular a contact transformation. Also biholomorphisms are known to be isometries of the Bergman metric $g_{1}$ (cf. e.g. [7], p. 370) hence symplectomorphisms of $\left(\Omega, \omega_{1}\right)$. On the other hand, one may weaken the assumption on $F$ by requesting only that $F$ be a $C^{\infty}$ diffeomorphism and $F^{*} \omega_{1}=\omega_{1}$. Then, by a result of A. Korányi and $\mathrm{H}$. M. Reimann [11], if $F$ is smooth up to the boundary then $F: \partial \Omega \rightarrow \partial \Omega$ is a contact transformation.

The main ingredient in the proof of A. Korányi and H. M. Reimann's result is the fact that, when $\gamma \equiv 1$, a certain negative power of the Bergman kernel $(\rho(z)=$ $\left.K_{1}(z, z)^{-1 /(n+1)}\right)$ is a defining function of $\Omega$ (allowing one to relate the symplectic structure of $\Omega$ to the contact structure of its boundary). In turn, this is a consequence of C. Fefferman's asymptotic expansion of $K_{1}(z, \zeta)$ (cf. Theorem 2 in

Received October 27, 1997 
[4], p. 9). Therefore, should one extend A. Korányi and H. M. Reimann's ideas to weighted Bergman kernels and related structures, the first obstacle is whether a similar asymptotic expansion is known for $K_{\gamma}(z, \zeta)$. Indeed, this is available when $\Omega=\{\varphi<0\}$ is a smoothly bounded strictly pseudoconvex domain and $\gamma=$ $|\varphi|^{m}, m \in\{0,1,2, \ldots\}$, by a result of M. M. Peloso [18] (cf. Theorem 1). Cf. also [19] for a study of the boundary behaviour of $K_{\gamma}(z, \zeta)$ when $\gamma=|\varphi|^{\alpha}, \alpha>-1$ (not necessarily an integer). However, each point of the curve $\alpha \mapsto|\varphi|^{\alpha}$ (in the Banach manifold $W(\Omega)$ of all weights on $\Omega$ ) is isolated (cf. Theorem 2 ) hence our present knowledge of the asymptoyic properties of $K_{\gamma}(z, \zeta)$, as $\gamma$ runs over $W(\Omega)$, is rather limited.

We apply Theorem 1 to study the boundary behaviour of a symplectomorphism of $\left(\Omega, \omega_{|\varphi|^{m}}\right), m \in\{1,2, \ldots\}$ (cf. Theorem 3 ).

Using the analytic behaviour of $K_{\gamma}(z, \zeta)$ with respect to $\gamma$ (cf. [16], p. 131) we prove an analogue of Fefferman's asymptotic formula for more general weights of the form: an essentially bounded function times a nonnegative integer power of the defining function (cf. Theorem 4).

In section 4 we show that the components of any symplectomorphism of a $\gamma$ Kobayashi domain $\Omega$ satisfy a Beltrami system (in the sense of [20]). If $\Omega$ is the Siegel domain, the tangential equations induced (on $\partial \Omega$ ) by this system turn out to be (cf. Proposition 2) the equations introduced in [10] in connection with the study of quasiconformal maps of strictly pseudoconvex CR manifolds (cf. also [9], [12]).

\section{The Forelli-Rudin-Ligocka-Peloso asymptotic expansion formula}

Let $\Omega \subset C^{n}$ be an open set and $W(\Omega)$ the set of all weights on $\Omega$ (i.e. $\gamma \in$ $W(\Omega)$ is a Lebesgue measurable function $\gamma: \Omega \rightarrow(0, \infty))$. For each $\gamma \in W(\Omega)$ let $L^{2}(\Omega, \gamma)$ be the Hilbert space of all functions $f: \Omega \rightarrow C$ for which

$$
\|f\|_{\gamma}=\left(\int_{\Omega}|f|^{2} \gamma d \mu\right)^{1 / 2}<\infty .
$$

Let $L^{2} H(\Omega, \gamma)$ be the set of all functions in $L^{2}(\Omega, \gamma)$ which are holomorphic in $\Omega$. A weight $\gamma \in W(\Omega)$ is admissible (cf. [17]) if 1) $L^{2} H(\Omega, \gamma)$ is a closed subspace of $L^{2}(\Omega, \gamma)$, and 2) for any $z \in \Omega$ the evaluation functional $\delta_{z}$ : $L^{2} H(\Omega, \gamma) \rightarrow C, \delta_{z}(f)=f(z)$, is continuous. The set of all admissible weights on $\Omega$ is denoted by $A W(\Omega)$. If $\gamma \in A W(\Omega)$ then, by the Riesz representation theorem, there is a unique function $K_{\gamma}(z, \cdot)$ (called the weighted Bergman kernel of $\Omega$, of weight $\gamma$, or the $\gamma$-Bergman kernel of $\Omega$ ) so that $\overline{K_{\gamma}(z, \cdot)} \in L^{2} H(\Omega, \gamma)$ and

$$
f(z)=\int_{\Omega} f(\zeta) K_{\gamma}(z, \zeta) \gamma(\zeta) d \mu(\zeta)
$$

for any $f \in L^{2} H(\Omega, \gamma), z \in \Omega$. For $\gamma=1$ this is the ordinary Bergman kernel of $\Omega$ (cf. e.g. [2]). 
Let $\Omega$ be a smoothly bounded strictly pseudoconvex domain $\Omega=\left\{z \in C^{n}\right.$ : $\varphi(z)<0\}$ where $\varphi$ is such that the Levi form $L_{\varphi}$ satisfies

$$
L_{\varphi}(w) \xi \geq C_{1}|\xi|^{2}, \quad \xi \in C^{n}
$$

for $\varphi(w)<\delta_{0}, \delta_{0}>0$, and $C_{1}$ depending only on $\Omega$. Set

$$
\Psi(\zeta, z)=(F(\zeta, z)-\varphi(z)) \chi(|\zeta-z|)+(1-\chi(|\zeta-z|))|\zeta-z|^{2}
$$

where

$$
F(\zeta, z)=-\sum_{J=1}^{n} \frac{\partial \varphi}{\partial z_{J}}(z)\left(\zeta_{J}-z_{J}\right)-\frac{1}{2} \sum_{J, k=1}^{n} \frac{\partial^{2} \varphi}{\partial z_{J} z_{k}}(z)\left(\zeta_{J}-z_{J}\right)\left(\zeta_{k}-z_{k}\right)
$$

and $\chi$ is a $C^{\infty}$ cut-off function of the real variable $t$, with $\chi(t)=1$ for $|t|<\varepsilon_{0} / 2$ and $\chi(t)=0$ for $|t| \geq 3 \varepsilon_{0} / 4$. We may state the following

THEOREM 1 (Forelli-Rudin-Ligocka-Peloso ${ }^{1}$ ). For any nonnegative integer $m \in\{0,1,2, \ldots\},|\varphi|^{m} \in A W(\Omega)$. Let $K_{m}(\zeta, z)$ be the $|\varphi|^{m}$-Bergman kernel for $L^{2} H\left(\Omega,|\varphi|^{m}\right)$. Then

$$
K_{m}(\zeta, z)=c_{\Omega}|\nabla \varphi(z)|^{2} \cdot \operatorname{det} L_{\varphi}(z) \cdot \Psi(\zeta, z)^{-(n+1+m)}+E(\zeta, z)
$$

where $E \in C^{\infty}(\bar{\Omega} \times \bar{\Omega}-\Delta), \Delta$ is the diagonal of $\partial \Omega \times \partial \Omega$, and $E$ satisfies the estimate

$$
|E(\zeta, z)| \leq c_{\Omega}^{\prime}|\Psi(\zeta, z)|^{-(n+1+m)+1 / 2} \cdot|\log | \Psi(\zeta, z)|| .
$$

This extends C. Fefferman's asymptotic expansion formula for the Bergman kernel of a strictly pseudoconvex domain (cf. [4] for $m=0$ ) to the case of $|\varphi|^{m}$ Bergman kernels, $m \in\{1,2, \ldots\}$ (cf. Lemma 2.2 in [18], p. 229). Part of the proof (relating $K_{m}(\zeta, z)$ to the ordinary Bergman kernel of the domain $\left.\left\{(z, \xi) \in C^{n} \times C^{m}: \varphi(z)+|\xi|^{2}<0\right\}\right)$ actually works for an arbitrary (admissible) weight. Indeed, one has the following

LEMMA 1. Let $m \in\{1,2, \ldots\}$ and $\gamma \in A W(\Omega)$. Let $K_{\Omega_{m}}((z, \xi),(w, \eta))$ be the Bergman kernel of the domain $\Omega_{m}=\left\{(z, \xi) \in \Omega \times C^{m}:|\xi|^{2 m}<\gamma(z)\right\}$. Then

$$
K_{\gamma}(z, w)=\frac{\omega_{2 m-1}}{2 m} K_{\Omega_{m}}((z, 0),(w, 0)) .
$$

Proof. For simplicity set $K(z, w)=K_{\Omega_{m}}((z, 0),(w, 0))$. Also, for fixed $z, w \in$ $\Omega$, we set $u(\eta)=K_{\Omega_{m}}((z, 0),(w, \eta))$. As $K_{\Omega_{m}}$ is anti-holomorphic in $\eta, u$ is

${ }^{1}$ We learned Theorem 1 from [18]. However, M. M. Peloso claims Theorem 1 is implicit in [14], while E. Ligocka employs an older idea by F Forelli and W. Rudin [6]. 
harmonic. Hence

$$
u(0)=\frac{2 m}{\omega_{2 m-1}} \gamma(w)^{-1} \int_{B\left(0, \gamma(w)^{1 /(2 m)}\right)} u(\eta) d \mu(\eta)
$$

where $\omega_{s}$ is the 'area' of the sphere $S^{s} \subset \boldsymbol{R}^{s+1}\left((w, \eta) \in \Omega_{m}\right.$ yields $\eta \in$ $\left.B\left(0, \gamma(w)^{1 /(2 m)}\right)\right)$. Therefore

$$
K(z, w) \gamma(w)=\frac{2 m}{\omega_{2 m-1}} \int_{|\eta|^{2 m}<\gamma(w)} K_{\Omega_{m}}((z, 0),(w, \eta)) d \mu(\eta) .
$$

For each $f \in L^{2} H(\Omega, \gamma)$ set $\tilde{f}(z, \xi)=f(z)$. Clearly $\tilde{f}$ is holomorphic in $\Omega_{m}$. Also

$$
\begin{aligned}
\|\tilde{f}\|_{L^{2}\left(\Omega_{m}\right)}^{2} & =\int_{\Omega_{m}}|\tilde{f}(z, \xi)|^{2} d \mu(z, \xi) \\
& =\int_{\Omega}|f(z)|^{2}\left(\int_{|\xi|^{2 m}<\gamma(z)} d \mu(\xi)\right) d \mu(z) \\
& =\frac{\omega_{2 m-1}}{2 m} \int_{\Omega}|f(z)|^{2} \gamma(z) d \mu(z)=\frac{\omega_{2 m-1}}{2 m}\|f\|_{\gamma}^{2}<\infty
\end{aligned}
$$

i.e. $\tilde{f} \in L^{2}\left(\Omega_{m}\right)$. As $K_{\Omega_{m}}$ reproduces the $L^{2}$ holomorphic functions on $\Omega_{m}$, one has (by (4))

$$
\begin{aligned}
f(z) & =\tilde{f}(z, 0)=\int_{\Omega_{m}} K_{\Omega_{m}}((z, 0),(w, \eta)) \tilde{f}(w, \eta) d \mu(w, \eta) \\
& =\int_{\Omega} f(w)\left(\int_{|\eta|^{2}<\gamma(w)} K_{\Omega_{m}}((z, 0),(w, \eta)) d \mu(\eta)\right) d \mu(w) \\
& =\frac{\omega_{2 m-1}}{2 m} \int_{\Omega} f(w) K(z, w) \gamma(w) d \mu(w),
\end{aligned}
$$

i.e. $\left(\omega_{2 m-1} / 2 m\right) K(z, w)$ reproduces the functions in $L^{2} H(\Omega, \gamma)$. As $u$ is antiholomorphic, $|u|^{2}$ is subharmonic. Hence

or

$$
|u(0)|^{2} \leq \frac{1}{\operatorname{Vol}\left(B\left(0, \gamma(w)^{1 /(2 m)}\right)\right)} \int_{B\left(0, \gamma(w)^{1 /(2 m)}\right)}|u(\eta)|^{2} d \mu(\eta)
$$

$$
|K(z, w)|^{2} \leq \frac{2 m}{\omega_{2 m-1}} \gamma(w)^{-1} \int_{|\eta|^{2}<\gamma(w)^{1 /(2 m)}}\left|K_{\Omega_{m}}((z, 0),(w, \eta))\right|^{2} d \mu(\eta) .
$$

Finally, we may integrate against $w \in \Omega$ so that to get

$$
\begin{aligned}
& \int_{\Omega}|K(z, w)|^{2} \gamma(w) d \mu(w) \\
& \quad \leq \frac{2 m}{\omega_{2 m-1}} \int_{\Omega_{m}}\left|K_{\Omega_{m}}((z, 0),(w, \eta))\right|^{2} d \mu(w, \eta)<\infty
\end{aligned}
$$


i.e. $K(z, \cdot) \in L^{2}(\Omega, \gamma)$. Then (3) follows from the uniqueness statement in the Riesz representation theorem.

When $\gamma=|\varphi|^{m}, m \in\{1,2, \ldots\}$, the domain $\Omega_{m}$ is strictly pseudoconvex and (2) follows from Lemma 1 and from Fefferman's asymptotic expansion formula for $K_{\Omega_{m}}$, i.e.

$$
\begin{aligned}
& K_{\Omega_{m}}((z, \xi),(w, \eta)) \\
& \quad=\text { const. }\left|\nabla \varphi_{1}(w, \eta)\right| \cdot \operatorname{det} L_{\varphi_{1}}(w, \eta) \cdot \Psi((z, \xi),(w, \eta))^{-(n+m+1)}+E((z, \xi),(w, \eta)),
\end{aligned}
$$

for some $E \in C^{\infty}\left(\bar{\Omega}_{m} \times \bar{\Omega}_{m}-\Delta_{1}\right)$ satisfying the estimate

$$
|E((z, \xi),(w, \eta))| \leq \text { const. }|\Psi((z, \xi),(w, \eta))|^{-(n+m+1)+1 / 2} \cdot|\log | \Psi((z, \xi),(w, \eta))||
$$

where $\Psi$ is defined as in (1), with the obvious modifications, while $\varphi_{1}(z, \xi)=$ $\varphi(z)+|\xi|^{2}$ and $\Delta_{1}$ is the diagonal of $\partial \Omega_{m} \times \partial \Omega_{m}$ (as $\partial \Omega \times\{0\} \subset \partial \Omega_{m}, \Delta$ imbeds in $\Delta_{1}$ ).

Let $L_{R}^{\infty}(\Omega)$ be the Banach space (algebra) of all real valued Lebesgue measurable, essentially bounded functions on $\Omega=\{\varphi<0\}$, with the norm $\|g\|_{\infty}=\operatorname{esssup}_{z \in \Omega}|g(z)|, g \in L_{R}^{\infty}(\Omega)$. By a result of Z. Pasternak-Winiarski (cf. Proposition 2.3 in [16], p. 116) $W(\Omega)$ is a Banach manifold modelled on $L_{R}^{\infty}(\Omega)$, and $A W(\Omega)$ is an open subset of $W(\Omega)$. Note that the Fefferman like asymptotic expansion of a weighted Bergman kernel is known (cf. Theorem 1 above) only for the points of the curve $C:(-1, \infty) \rightarrow W(\Omega), C(\alpha)=$ $|\varphi|^{\alpha} \in A W(\Omega), \alpha>-1$, corresponding to the integer values of the parameter. Of course, it is desirable to extend Theorem 1 to all $\gamma \in A W(\Omega)$. As a measure of the amount of job left unsolved we may state the following

THEOREM 2. Let $\Omega=\{\varphi<0\}$ be a domain in $C^{n}$. The curve $C:(-1, \infty)$ $\rightarrow W(\Omega), C(\alpha)=|\varphi|^{\alpha}, \alpha>-1$, is discontinuous and each point of $C$ is an isolated point.

Set

$$
U(\Omega)=\left\{g \in L_{\boldsymbol{R}}^{\infty}(\Omega): \operatorname{essinf}_{z \in \Omega} g(z)>0\right\}
$$

(an open subset of $L_{R}^{\infty}(\Omega)$ ). Given $\mu \in W(\Omega)$ let $\Phi_{\mu}: U(\Omega) \rightarrow W(\Omega)$ be defined by $\left(\Phi_{\mu} g\right)(z)=g(z) \mu(z), g \in U(\Omega), \quad z \in \Omega$, and set $U(\Omega, \mu)=\Phi_{\mu}(U(\Omega))$. By Proposition 2.3 in [16], p. 116, the family

$$
\left\{\Phi_{\mu}(A): \mu \in W(\Omega), A \subseteq U(\Omega), A \text { open }\right\}
$$

is a basis of open sets for the topology of $W(\Omega)$. At this point, we may prove Theorem 2. Given $\alpha_{0}>-1, C$ is continuous in $\alpha_{0}$ if and only if for any open subset $A \subseteq U(\Omega)$ with $1 \in A$, there is $\delta_{A}>0$ so that $|\varphi|^{\alpha-\alpha_{0}} \in A$ for any $\left|\alpha-\alpha_{0}\right|<\delta_{A}$. Note that for each $u: \bar{\Omega} \rightarrow[0, \infty)$, if $u \in C^{0}(\bar{\Omega})$ and $\left.u\right|_{\partial \Omega}=0$ then $\operatorname{essinf}_{\Omega} u \leq 0$ (indeed, if $\operatorname{essinf}_{\Omega} u>0$ then

$$
u(z) \geq L
$$


for some $L>0$. A priori (5) holds a.e. in $\Omega$, yet $\{u<L\}$ is open, hence empty. Therefore (5) holds everywhere in $\Omega$ and, for $z \rightarrow \partial \Omega$, it gives $L \leq 0$, a contradiction).

Lemma 2. Let $\alpha_{0}>-1, \delta>0$ and $A$ an open subset of $U(\Omega)$ with $1 \in A$. Then $|\varphi|^{\alpha-\alpha_{0}} \in A$ if and only if $\alpha=\alpha_{0}$.

Proof. If $\alpha>\alpha_{0}$ then (by the observation above) $\left.|\varphi|^{\alpha-\alpha_{0}}\right|_{\partial \Omega}=0$ yields $|\varphi|^{\alpha-\alpha_{0}} \notin U(\Omega)$. If in turn $\alpha<\alpha_{0}$ then $\lim _{z \rightarrow \partial \Omega}|\varphi(z)|^{\alpha-\alpha_{0}}=\infty$ hence $|\varphi|^{\alpha-\alpha_{0}} \notin$ $L_{R}^{\infty}(\Omega)$, just by observing that, for each $v: \Omega \rightarrow[0, \infty)$, if $v \in C^{0}(\Omega)$ and $\lim _{z \rightarrow \partial \Omega} v(z)=\infty$ then $\operatorname{esssup}_{\Omega} v=\infty$.

Finally $U\left(\Omega,|\varphi|^{\alpha_{0}}\right)$ is an open neighborhood of $|\varphi|^{\alpha_{0}}$ yet (by Lemma 2) it contains no other point of $C$.

\section{Symplectomorphisms of $\gamma$-Kobayashi domains}

Let $\Omega=\{\varphi<0\}$ be a domain and $\gamma \in A W(\Omega)$ and admissible weight. By a result in [17] one has the representation

$$
K_{\gamma}(\zeta, z)=\sum_{k} \phi_{k}(\zeta) \overline{\phi_{k}(z)}
$$

for any complete orthonormal system $\left\{\phi_{k}\right\}$ in $L^{2} H(\Omega, \gamma)$. Hence $K_{\gamma}(z, z)>0$ for any $z \in \Omega$, provided that A) for each $z \in \Omega$ there is $f \in L^{2} H(\Omega, \gamma)$ with $f(z)$ $\neq 0$. If the weight $\gamma=(1+h)|\varphi|^{m}$ (with $h \in L_{\boldsymbol{R}}^{\infty}(\Omega),\|h\|_{\infty}<1 / 2, m \in\{1,2, \ldots\}$ ) satisfies condition $\mathrm{A})$ then it makes sense to consider the function

$$
\rho_{h, m}(z)=K_{(1+h)|\varphi|^{m}}(z, z)^{-1 /(n+1+m)}, \quad z \in \Omega,
$$

and (by Theorem 4)

$$
\begin{aligned}
& \rho_{h, m}(z) \\
& \quad \leq|\varphi(z)|\left\{\Phi(z)+C\left[|\varphi(z)|^{1 / 2}|\log | \varphi(z)||+(1+F(z))^{2}\right]\right\}^{-1 /(n+1+m)}
\end{aligned}
$$

for some $\Phi \in C^{\infty}(\bar{\Omega})$ so that $\Phi(z) \neq 0$ near $\partial \Omega$. Hence $\rho_{h, m}(z) \rightarrow 0$ as $z \rightarrow \partial \Omega$. As the boundary behaviour of $\left[K_{1,|\varphi|^{m}}^{(k)} h^{(k)}\right](z, w), k \geq 1$ (cf. notations in section 3) is not known, one may not conclude that $\rho_{h, m}(z)$ is a defining function for $\Omega$. However, as a corollary of Theorem 1 one has

$$
K_{m}(z, z)=\Phi(z)|\varphi(z)|^{-(n+1+m)}+\tilde{\Phi}(z) \log |\varphi(z)|,
$$

for some $\Phi, \tilde{\Phi} \in C^{\infty}(\bar{\Omega}), \Phi(z) \neq 0$ near $\partial \Omega$, hence $\rho_{m}=\rho_{0, m} \in C^{\infty}(\bar{\Omega})$ and $\nabla \rho_{m} \neq 0$ on $\partial \Omega$, i.e. $\rho_{m}$ can be used as a defining function for $\Omega\left(\Omega=\left\{\rho_{m}>0\right\}\right)$.

Let $\Omega_{n}=\left\{\zeta \in C^{n}: \varphi_{n}(\zeta)<0\right\}$ be the Siegel domain, where $\varphi_{n}(\zeta)=\left|\zeta^{\prime}\right|^{2}-$ $\operatorname{Im}\left(\zeta_{1}\right)$, and for each $\zeta=\left(\zeta_{1}, \ldots, \zeta_{n}\right)$ one sets $\zeta^{\prime}=\left(\zeta_{2}, \ldots, \zeta_{n}\right)$. Let $K_{\alpha}(\zeta, z)$ be 
the $\left|\varphi_{n}\right|^{\alpha}$-Bergman kernel for $L^{2} H\left(\Omega_{n},\left|\varphi_{n}\right|^{\alpha}\right), \alpha>-1$. As $\Omega_{n}$ is unbounded and $\alpha$ not necessarily an integer, neither Theorem 1 nor its proof apply, yet $\rho_{\alpha}(\zeta)=$ $K_{\alpha}(\zeta, \zeta)^{-1 /(n+1+\alpha)}$ is a (well defined) defining function for $\Omega_{n}$. Indeed (cf. [1]) $K_{\alpha}$ may be explicitely computed as

$$
\begin{aligned}
K_{\alpha}(\zeta, z) & =\frac{2^{n-1+\alpha} c_{n, \alpha}}{\left[i\left(\bar{z}_{1}-\zeta_{1}\right)-2\left\langle\zeta^{\prime}, z^{\prime}\right\rangle\right]^{n+1+\alpha}} \\
c_{n, \alpha} & =\pi^{-n}(\alpha+1) \cdots(\alpha+n)
\end{aligned}
$$

hence $\rho_{\alpha}(\zeta)=C \varphi_{n}(\zeta)$, for some constant $C$ depending only on $n$ and $\alpha$.

Let $\Omega \subset C^{n}$ be a domain and $\gamma \in A W(\Omega)$. In general $g_{\gamma}$ is not definite, or even nondegenerate. For instance, if $\Omega$ is bounded and $\gamma \in L^{1}(\Omega)$ then $g_{\gamma}$ is a Kählerian metric on $\Omega$ (cf. [3]) yet the arguments in [3] break down for the case of an unbounded domain. We call $\Omega$ a $\gamma$-Kobayashi domain if $(\Omega, \gamma)$ satisfies condition A) and additionally B) for any $z \in \Omega$ and any $Z \in T^{1,0}(\Omega)_{z}, Z \neq 0$, there is $f \in L^{2} H(\Omega, \gamma)$ so that $f(z)=0$ and $Z(f) \neq 0$ (our $\mathrm{A}$ )-B) correspond to the conditions (A.1)-(A.2) in [8], pp. 271-272, hence the adopted terminology). Here $T^{1,0}(\Omega)$ is the holomorphic tangent bundle over $\Omega$. The unit ball in $\boldsymbol{C}^{n}$ is a 1-Kobayashi domain. The Siegel domain $\Omega_{n}$ is an (unbounded) $\left|\varphi_{n}\right|^{\alpha}$ Kobayashi domain for any $\alpha>-1$ (cf. Lemmae 4 and 5 in [1]). By a result in [15], A)-B) imply that $g_{\gamma}$ is a Kählerian metric on $\Omega$, hence $\left(\Omega, \omega_{\gamma}\right)$ is a symplectic manifold.

From now on, it is understood that $\Omega$ is a strictly pseudoconvex domain satisfying all hypothesis of Theorem 1 . We may state:

THEOREM 3. Let $m \in\{0,1,2, \ldots\}$ and $\Omega=\{\varphi<0\}$ a $|\varphi|^{m}$-Kobayashi domain. Let $F$ be a symplectomorphism of $\left(\Omega, \omega_{m}\right)$, i.e. a $C^{\infty}$ diffeomorphism $F$ : $\Omega \rightarrow \Omega$ with $F^{*} \omega_{m}=\omega_{m}$. If $F$ is smooth up to the boundary then $F: \partial \Omega \rightarrow \partial \Omega$ is a contact transformation.

Here $\omega_{m}$ is short for $\omega_{|\varphi|^{m}}$. For $\gamma=1$ and $m=0$ Theorem 3 is the result by A. Korányi and H. M. Reimann quoted in the introduction. The proof is imitative of that of Proposition 1 in [11], p. 1121. We need some notation. Let $\mathscr{F}$ be the foliation of $U$ (a one-sided neighborhood of the boundary of $\Omega$ ) by level sets of $\rho_{m}$ (so that $\rho_{m}^{-1}(0)=\partial \Omega$ ). Each leaf $M_{c}=\rho_{m}^{-1}(c)$ is a strictly pseudoconvex CR manifold with the CR structure $T_{1,0}\left(M_{c}\right)=\left[T\left(M_{c}\right) \otimes C\right] \cap$ $T^{1,0}(U)$. Let $T_{1,0}(\mathscr{F})$ be the subbundle of $T(U) \otimes C$ whose portion over $M_{c}$ is $T_{1,0}\left(M_{c}\right)$. As $\Omega$ is strictly pseudoconvex, there is a uniquely defined complex vector field $\xi$ of type $(1,0)$ on $U$ which is orthogonal to $T_{1,0}(\mathscr{F})$ with respect to $\partial \bar{\partial} \rho_{m}$ and for which $\partial \rho_{m}(\xi)=1$ (cf. e.g. [13], p. 163). Define $r: U \rightarrow \boldsymbol{R}$ by setting $r=2\left(\partial \bar{\partial} \rho_{m}\right)(\xi, \bar{\xi})$ so that $\xi$ and $r$ are characterized by

$$
\xi\rfloor \partial \bar{\partial} \rho_{m}=r \bar{\partial} \rho_{m}, \quad \partial \rho_{m}(\xi)=1 .
$$

Let $\theta_{m}=i(\bar{\partial}-\partial) \rho_{m} / 2$ and $N=2 \operatorname{Re}(\xi)$. Then $\left(d \rho_{m}\right) N=2$ and $\theta_{m}(N)=0$. 
Note that

$$
\omega_{m}=i(n+1+m)\left(\frac{\partial \bar{\partial} \rho_{m}}{\rho_{m}}-\frac{\partial \rho_{m} \wedge \bar{\partial} \rho_{m}}{\rho_{m}^{2}}\right) .
$$

Set $H(\mathscr{F})=\operatorname{Re}\left\{T_{1,0}(\mathscr{F}) \oplus \overline{T_{1,0}(\mathscr{F})}\right\}$ (so that the portion of $H(\mathscr{F})$ over a leaf $M_{c}$ is the maximally complex, or Levi, distribution of $M_{c}$ ). Then (by (7))

$$
\omega_{m}(X, N)=0
$$

for any $X \in H(\mathscr{F})$. On the other hand, we may write (7) as

$$
\omega_{m}=(n+1+m)\left(\frac{d \theta_{m}}{\rho_{m}}-\frac{d \rho m \wedge \theta_{m}}{\rho_{m}^{2}}\right)
$$

hence (by $F^{*} \omega_{m}=\omega_{m}$ )

$$
\begin{aligned}
0= & \omega_{m}((d F) X,(d F) N)=(n+1+m) \rho_{m}^{-1} d \theta_{m}((d F) X,(d F) N) \\
& -(n+1+m) \rho_{m}^{-2}\left(d \rho_{m} \wedge \theta_{m}\right)((d F) X,(d F) N)
\end{aligned}
$$

for any $X \in H(\mathscr{F})$. As $F$ is smooth up to the boundary,

$$
\left(d \theta_{m}\right)((d F) X,(d F) N)
$$

stays finite near $\partial \Omega$. Hence, in the limit

$$
\left(d \rho_{m}\right)((d F) X) \theta_{m}((d F) N)-\left(d \rho_{m}\right)((d F) N) \theta_{m}((d F) X)
$$

vanishes on $\partial \Omega$. If $X$ lies in $H(\partial \Omega)$, the maximal complex distribution of $\partial \Omega$ as a CR manifold, then $(d F) X \in T(\partial \Omega)$ hence $\left(d \rho_{m}\right)((d F) X)=0$. Finally $\left(d \rho_{m}\right)$ $((d F) N) \neq 0$ (as $F$ is a diffeomorphism and $d \rho_{m} \neq 0$ on $\partial \Omega$ ) hence $\theta_{m}((d F) X)=$ 0 for any $X \in H(\partial \Omega)$.

q.e.d.

Let $\omega_{\alpha}$ be short for $\omega_{\left|\varphi_{n}\right|^{\alpha}}, \alpha>-1$. Although $\Omega_{n}$ is unbounded and $\alpha$ not necessarily an integer, Theorem 3 remains true for a symplectomorphism $F$ of $\left(\Omega_{n}, \omega_{\alpha}\right)$, i.e. if $F$ is smooth up to $\partial \Omega_{n}$ then the restriction of $F$ to $\partial \Omega_{n}$ is a contact transformation (the proof is a verbatim transcription of the proof of Theorem 3 , where $\rho_{m}$ is replaced by $\rho_{\alpha}$ ).

\section{The effect of the analytic behaviour of weighted Bergman kernels}

Let $U$ be an open subset of a normed space $\mathscr{X}$ and let $\mathscr{Y}$ be a topological vector space. Together with [16], one says that a map $f: U \rightarrow \mathscr{Y}$ is analytic on $U$ if for any $x \in U$ there is a ball $B \subset \mathscr{X}$ of center $0 \in \mathscr{X}$ so that $x+B \subset U$ and

$$
f(x+h)=f(x)+\sum_{k=1}^{\infty} a_{k}(h, \ldots, h)
$$

for any $h \in B$, where $a_{k}: \mathscr{X}^{k} \rightarrow \mathscr{Y}$ is a continuous $k$-linear function, $k \in\{1,2, \ldots\}$, and the series in (8) converges uniformly on $B$. 
Let $H A(\Omega)$ be the vector space of all real analytic functions $F: \Omega \times \Omega \rightarrow C$ which are holomorphic with respect to the first $n$ variables and anti-holomorphic with respect to the last $n$ variables. Set

$$
\|F\|_{X}=\sup _{(z, \zeta) \in X^{2}}|F(z, \zeta)|
$$

for $F \in H A(\Omega), X \subset \Omega$. The family of seminorms

$$
\left\{\|\cdot\|_{X}: X \subset \Omega, X \text { compact }\right\}
$$

makes $H A(\Omega)$ into a Fréchet space. By a result of $Z$. Pasternak-Winiarski (cf. Theorem 5.1 in [16], p. 131) the map $U(\Omega) \rightarrow H A(\Omega), g \mapsto K_{g \gamma}$, is analytic on $U(\Omega)$ for any $\gamma \in A W(\Omega)$.

THEOREM 4. Let $\Omega=\{\varphi<0\}$ be a smoothly bounded strictly pseudoconvex domain in $\boldsymbol{C}^{n}$ so that $L_{\varphi}(w) \xi \geq$ const. $|\xi|^{2}, \xi \in \boldsymbol{C}^{n}$, for $\varphi(w)<\delta_{0}, \delta_{0}>0$. Then for any $h \in B(0,1 / 2) \subset L_{\boldsymbol{R}}^{\infty}(\Omega)$ there is $E_{h} \in C^{\infty}(\Omega \times \Omega)$ so that

$$
K_{(1+h)|\varphi|^{m}}(z, w)=c_{\Omega}|\nabla \varphi(w)|^{2} \cdot \operatorname{det} L_{\varphi}(w) \cdot \Psi(z, w)^{-(n+1+m)}+E_{h}(z, w)
$$

and $E_{h}$ satisfies the estimate

$$
\begin{aligned}
\left(E_{h}(z, w) \mid \leq\right. & C \cdot\left\{|\Psi(z, w)|^{-(n+1+m)+1 / 2}|\log | \Psi(z, w) \|\right. \\
& \left.+|\varphi(z)|^{-(n+1+m) / 2}|\varphi(w)|^{-(n+1+m) / 2}(1+F(z)+F(w)+F(z) F(w))\right\}
\end{aligned}
$$

where $F(z)=|\varphi(z)|^{3 / 2}+|\varphi(z)|^{1 / 2}|\log | \varphi(z) \|$ and $C$ is a constant depending only on $\Omega$ and $m \geq 1, m>n-1$.

The proof of Theorem 4 relies on (2) and on the analyticity of the weighted Bergman kernel as a map $A W(\Omega) \rightarrow H A(\Omega), \gamma \mapsto K_{\gamma}$. Set

$$
\begin{aligned}
{\left[K_{g, \gamma}^{(k)}\left(h_{1}, \ldots, h_{k}\right)\right](z, w) } & \\
= & \int_{\Omega} K_{g \gamma}\left(u_{1}, w\right) h_{1}\left(u_{1}\right) \gamma\left(u_{1}\right) d \mu\left(u_{1}\right) \\
& \cdot \int_{\Omega} K_{g \gamma}\left(u_{2}, u_{1}\right) h_{2}\left(u_{2}\right) \gamma\left(u_{2}\right) d \mu\left(u_{2}\right) \ldots \\
& \cdot \int_{\Omega} K_{g \gamma}\left(u_{k}, u_{k-1}\right) h_{k}\left(u_{k}\right) K_{g \gamma}\left(z, u_{k}\right) \gamma\left(u_{k}\right) d \mu\left(u_{k}\right)
\end{aligned}
$$

for $\gamma \in A W(\Omega), g \in U(\Omega), h_{1}, \ldots, h_{k} \in L_{R}^{\infty}(\Omega), k \geq 1$. Then

$$
K_{g, \gamma}^{(k)}\left(h_{1}, \ldots, h_{k}\right) \in H A(\Omega)
$$

(cf. Lemma 5.1 in [16], p. 129). By (2) and by (5.5) in Theorem 5.1 of [16], p. 131 , it follows that (9) holds good with 


$$
E_{h}=E+\sum_{k=1}^{\infty}(-1)^{k} K_{1,|\varphi|^{m}}^{(k)} h^{(k)}
$$

where $h^{(k)}=(h, \ldots, h)$ ( $k$ components), $E \in C^{\infty}(\bar{\Omega} \times \bar{\Omega}-\Delta)$ satisfies the estimate in Theorem 1, and the series is uniformly convergent on $B(0,1 / 2)=\{h \in$ $\left.L_{R}^{\infty}(\Omega):\|h\|_{\infty}<1 / 2\right\}$ with respect to any seminorm $\|\cdot\|_{X}$ on $H A(\Omega)$, with $X$ an arbitrary compact subset of $\Omega$. It remains that we prove the estimate (10). Let $k \geq 3$ (the cases $k=1$ and $k=2$ are looked at later on). Then (by (2))

$$
\begin{aligned}
{\left[K_{1,|\varphi|^{m}}^{(k)} h^{(k)}\right](z, w) } & \\
= & \int\left\{c_{\Omega}\left|\nabla \varphi\left(u_{1}\right)\right|^{2} \cdot \operatorname{det} L_{\varphi}\left(u_{1}\right) \cdot \overline{\Psi\left(w, u_{1}\right)}-(n+1+m)\right. \\
& \left.\cdot \overline{E\left(w, u_{1}\right)}\right\} \\
& \cdot\left\{c_{\Omega}\left|\nabla \varphi\left(u_{k}\right)\right|^{2} \cdot \operatorname{det} L_{\varphi}\left(u_{k}\right) \cdot \Psi\left(z, u_{k}\right)^{-(n+1+m)}+E\left(z, u_{k}\right)\right\} \\
& \cdot h\left(u_{1}\right) h\left(u_{k}\right)\left[K_{1,|\varphi|^{m}}^{(k-2)} h^{(k-2)}\right]\left(u_{k}, u_{1}\right)\left|\varphi\left(u_{1}\right)\right|^{m}\left|\varphi\left(u_{k}\right)\right|^{m} d \mu\left(u_{1}\right) d \mu\left(u_{k}\right)
\end{aligned}
$$

hence

$$
\begin{aligned}
& {\left[K_{1,|\varphi|^{m}}^{(k)} h^{(k)}\right](z, w)} \\
& \quad=c_{\Omega}^{2} I_{1}(z, w)+c_{\Omega}\left(I_{2}(z, w)+I_{3}(z, w)\right)+I_{4}(z, w),
\end{aligned}
$$

where

$$
\begin{aligned}
I_{j}(z, w)= & \int_{\Omega} G_{j}\left(u_{1}, w\right)\left(\int_{\Omega}\left[K_{1,|\varphi|^{m}}^{(k-2)} h^{(k-2)}\right]\left(u_{k}, u_{1}\right) H_{j}\left(z, u_{k}\right)\left|\varphi\left(u_{k}\right)\right|^{m} d \mu\left(u_{k}\right)\right) \\
& \cdot\left|\varphi\left(u_{1}\right)\right|^{m} d \mu\left(u_{1}\right)
\end{aligned}
$$

for $1 \leq j \leq 4$ and $G_{j}, H_{j}$ are given by

$$
\begin{aligned}
& G_{1}\left(u_{1}, w\right)=G_{2}\left(u_{1}, w\right)=\left|\nabla \varphi\left(u_{1}\right)\right|^{2} \cdot \operatorname{det} L_{\varphi}\left(u_{1}\right) \cdot \overline{\Psi\left(w, u_{1}\right)} \\
& G_{3}\left(u_{1}, w\right)=G_{4}\left(u_{1}, w\right)=\overline{E\left(w, u_{1}\right)} h\left(u_{1}\right) \\
& H_{1}\left(z, u_{k}\right)=H_{3}\left(z, u_{k}\right)=\left|\nabla \varphi\left(u_{k}\right)\right|^{2} \cdot \operatorname{det} L_{\varphi}\left(u_{k}\right) \cdot \Psi\left(z, u_{k}\right)^{-(n+1+m)} h\left(u_{k}\right) \\
& H_{2}\left(z, u_{k}\right)=H_{4}\left(z, u_{k}\right)=E\left(z, u_{k}\right) h\left(u_{k}\right) .
\end{aligned}
$$

By a result in [16], p. 131, we have

$$
\left\|\left[K_{1,|\varphi|^{m}}^{(k-2)} h^{(k-2)}\right]\left(\cdot, u_{1}\right)\right\|_{m} \leq\|h\|_{\infty}^{k-2}\left\|K_{m}\left(\cdot, u_{1}\right)\right\|_{m}
$$

where $\|\cdot\|_{m}$ is short for $\|\cdot\|_{|\varphi|^{m}}$. Then we may perform the estimates 


$$
\begin{aligned}
\left|I_{j}(z, w)\right| \leq & \int_{\Omega}\left|G_{j}\left(u_{1}, w\right)\right| \\
& \cdot\left(\int_{\Omega}\left|\left[K_{1,|\varphi|^{m}}^{(k-2)} h^{(k-2)}\right]\left(u_{k}, u_{1}\right)\right|^{2}\left|\varphi\left(u_{k}\right)\right|^{m} d \mu\left(u_{k}\right)\right)^{1 / 2} \\
& \cdot\left(\int_{\Omega}\left|H_{j}\left(z, u_{k}\right)\right|^{2} \cdot\left|\varphi\left(u_{k}\right)\right|^{m} d \mu\left(u_{k}\right)\right)^{1 / 2}\left|\varphi\left(u_{1}\right)\right|^{m} d \mu\left(u_{1}\right) \\
= & \int_{\Omega}\left|G_{j}\left(u_{1}, w\right)\right| \cdot\left\|\left[K_{1,|\varphi|^{m}}^{(k-2)} h^{(k-2)}\right]\left(\cdot, u_{1}\right)\right\|_{m} \cdot\left\|H_{j}(z, \cdot)\right\|_{m}\left|\varphi\left(u_{1}\right)\right|^{m} d \mu\left(u_{1}\right) \\
\leq & \|h\|_{\infty}^{k-2}\left\|H_{j}(z, \cdot)\right\|_{m} \cdot \int_{\Omega}\left|G_{j}\left(u_{1}, w\right)\right| \cdot\left\|K_{m}\left(\cdot, u_{1}\right)\right\|_{m}\left|\varphi\left(u_{1}\right)\right|^{m} d \mu\left(u_{1}\right) .
\end{aligned}
$$

Yet

$$
\left\|K_{m}\left(\cdot, u_{1}\right)\right\|_{m} \leq \text { const. }\left|\varphi\left(u_{1}\right)\right|^{-(n+1+m) / 2}
$$

by Lemma 2.8 in [18], p. 233 . Hence

$$
\begin{aligned}
\left|I_{j}(z, w)\right| \leq \text { const. }\|h\|_{\infty}^{k-2}\left\|H_{j}(z, \cdot)\right\|_{m} \\
\cdot \int_{\Omega}\left|G_{j}\left(u_{1}, w\right)\right| \cdot\left|\varphi\left(u_{1}\right)\right|^{-(n+1-m) / 2} d \mu\left(u_{1}\right) .
\end{aligned}
$$

We look at the case $j=1$. To this end, set

$$
J_{v, a}(z)=\int_{\Omega} \frac{|\varphi(w)|^{v} d \mu(w)}{|\Psi(z, w)|^{n+1+v+a}}
$$

for $v>-1$ and $a \in \boldsymbol{R}$. By Lemma 2.7 in [18], p. 232, one has

$$
J_{v, a}(z) \leq \begin{cases}\text { const. } & \text { if } a<0 \\ |\log | \varphi(z)|| & \text { if } a=0 \\ |\varphi(z)|^{-a} & \text { if } a>0\end{cases}
$$

Then

$$
\begin{aligned}
\left\|H_{1}(z, \cdot)\right\|_{m}^{2} & =\int_{\Omega}\left|H_{1}\left(z, u_{k}\right)\right|^{2}\left|\varphi\left(u_{k}\right)\right|^{m} d \mu\left(u_{k}\right) \\
& \leq \text { const. }\|h\|_{\infty}^{2} \int_{\Omega}\left|\Psi\left(z, u_{k}\right)\right|^{-2(n+1+m)}\left|\varphi\left(u_{k}\right)^{m}\right| d \mu\left(u_{k}\right) \\
& =\text { const. }\|h\|_{\infty}^{2} J_{m, n+1+m}(z)
\end{aligned}
$$

so that

$$
\left\|H_{1}(z, \cdot)\right\|_{m} \leq \text { const. }\|h\|_{\infty}|\varphi(z)|^{-(n+1+m) / 2}
$$


Then (by (11))

$$
\begin{aligned}
&\left|I_{1}(z, w)\right| \leq \text { const. }\|h\|_{\infty}^{k-1}|\varphi(z)|^{-(n+1+m) / 2} \cdot \int_{\Omega}\left|G_{1}\left(u_{1}, w\right)\right|\left|\varphi\left(u_{1}\right)\right|^{-(n+1+m) / 2} d \mu\left(u_{1}\right) \\
& \leq \text { const. }\|h\|_{\infty}^{k}|\varphi(z)|^{-(n+1+m) / 2} \int_{\Omega} \frac{\left|\varphi\left(u_{1}\right)\right|^{-(n+1-m) / 2} d \mu\left(u_{1}\right)}{\left|\Psi\left(w, u_{1}\right)\right|^{n+1+m}} \\
&=\text { const. }\|h\|_{\infty}^{k}|\varphi(z)|^{-(n+1+m) / 2} J_{-(n+1-m) / 2,(n+1+m) / 2}(w) .
\end{aligned}
$$

We may conclude that

$$
\left|I_{1}(z, w)\right| \leq \text { const. }\|h\|_{\infty}^{k}|\varphi(z)|^{-(n+1+m) / 2}|\varphi(w)|^{-(n+1+m) / 2} .
$$

Next $($ for $j=2$ )

$$
\begin{aligned}
\left\|H_{2}(z, \cdot)\right\|_{m}^{2} & =\int_{\Omega}\left|E\left(z, u_{k}\right)\right|^{2}\left|h\left(u_{k}\right)\right|^{2}\left|\varphi\left(u_{k}\right)\right|^{m} d \mu\left(u_{k}\right) \\
& \leq \text { const. }\|h\|_{\infty}^{2} \int_{\Omega}\left|\Psi\left(z, u_{k}\right)\right|^{-2(n+1+m)+1}|\log | \Psi\left(z, u_{k}\right) \|^{2}\left|\varphi\left(u_{k}\right)\right|^{m} d \mu\left(u_{k}\right) .
\end{aligned}
$$

This integral may be written as a sum $\int_{\left\{\left|\Psi\left(z, u_{k}\right)\right| \geq 1\right\}}+\int_{\left\{\left|\Psi\left(z, u_{k}\right)\right|<1\right\}}$. In the first integral $\log \left|\Psi\left(z, u_{k}\right)\right| \leq\left|\Psi\left(z, u_{k}\right)\right|$ while for the second (cf. [18], p. 229)

$$
\begin{aligned}
\left|\Psi\left(z, u_{k}\right)\right| & \geq \text { const. }\left(|\varphi(z)|+\left|\varphi\left(u_{k}\right)\right|+\left|z-u_{k}\right|^{2}+\left|\operatorname{Im} \Psi\left(z, u_{k}\right)\right|\right) \\
& \geq \text { const. }|\varphi(z)|
\end{aligned}
$$

yields $|\log | \Psi\left(z, u_{k}\right) \| \leq$ const. $|\log | \varphi(z) \|$. Hence

$$
\begin{aligned}
\left\|H_{2}(z, \cdot)\right\|_{m}^{2} \leq & \text { const. }\|h\|_{\infty}^{2} \\
& \cdot\left(\int_{\Omega}\left|\Psi\left(z, u_{k}\right)\right|^{-2(n+m)+1}\left|\varphi\left(u_{k}\right)\right|^{m} d \mu\left(u_{k}\right)\right. \\
& \left.+ \text { const. }|\log | \varphi(z) \|^{2} \int_{\Omega}\left|\Psi\left(z, u_{k}\right)\right|^{-2(n+m)-1}\left|\varphi\left(u_{k}\right)\right|^{m} d \mu\left(u_{k}\right)\right) \\
= & \text { const. } \left.\|h\|_{\infty}^{2}\left(J_{m, n-2+m}(z)+|\log | \varphi(z)\right) \|^{2} J_{m, n+m}(z)\right)
\end{aligned}
$$

i.e.

$$
\left\|H_{2}(z, \cdot)\right\|_{m} \leq \text { const. }\|h\|_{\infty}|\varphi(z)|^{-(n+m) / 2}(|\varphi(z)|+|\log | \varphi(z) \|) .
$$

Then (by (11)) 


$$
\begin{aligned}
\left|I_{2}(z, w)\right| \leq & \text { const. }\|h\|_{\infty}^{k-1}|\varphi(z)|^{-(n+m) / 2}(|\varphi(z)|+|\log | \varphi(z) \|) \\
& \cdot \int_{\Omega}\left|G_{2}\left(u_{1}, w\right)\right|\left|\varphi\left(u_{1}\right)\right|^{-(n+1-m) / 2} d \mu\left(u_{1}\right) \\
\leq & \text { const. }\|h\|_{\infty}^{k}|\varphi(z)|^{-(n+m) / 2}(|\varphi(z)| \\
& +|\log | \varphi(z) \|) \int_{\Omega} \frac{\left|\varphi\left(u_{1}\right)\right|^{-(n+1-m) / 2} d \mu\left(u_{1}\right)}{\left|\Psi\left(w, u_{1}\right)\right|^{n+1+m}} \\
= & \text { const. }\|h\|_{\infty}^{k}|\varphi(z)|^{-(n+m) / 2}(|\varphi(z)+| \log \mid \varphi(z) \|) J_{-(n+1-m) / 2,(n+1+m) / 2}(w)
\end{aligned}
$$

i.e.

$$
\left|I_{2}(z, w)\right| \leq \text { const. }\|h\|_{\infty}^{k}|\varphi(z)|^{-(n+1+m) / 2}|\varphi(w)|^{-(n+1+m) / 2} F(z) .
$$

Next (as $\left.H_{1}=H_{3}\right)$

$$
\begin{aligned}
\left|I_{3}(z, w)\right| \leq & \text { const. }\|h\|_{\infty}^{k}|\varphi(z)|^{-(n+1+m) / 2} \\
& \cdot \int_{\Omega}\left|E\left(w, u_{1}\right)\right| \cdot\left|\varphi\left(u_{1}\right)\right|^{-(n+1-m) / 2} d \mu\left(u_{1}\right) \\
\leq & \text { const. }\|h\|_{\infty}^{k}|\varphi(z)|^{-(n+1+m) / 2} \int_{\Omega}\left|\Psi\left(w, u_{1}\right)\right|^{-(n+1+m)+1 / 2} \\
& \cdot|\log | \Psi\left(w, u_{1}\right) \| \cdot\left|\varphi\left(u_{1}\right)\right|^{-(n+1-m) / 2} d \mu\left(u_{1}\right) \\
\leq & \text { const. }\|h\|_{\infty}^{k}|\varphi(z)|^{-(n+1+m) / 2} \\
& \cdot\left\{\int_{\Omega}\left|\Psi\left(w, u_{1}\right)\right|^{-(n+m)+1 / 2} \cdot\left|\varphi\left(u_{1}\right)\right|^{-(n+1-m) / 2} d \mu\left(u_{1}\right)\right. \\
& \left.+ \text { const. }|\log | \varphi(w) \| \int_{\Omega}\left|\Psi\left(w, u_{1}\right)\right|^{-(n+m)-1 / 2}\left|\varphi\left(u_{1}\right)\right|^{-(n+1-m) / 2} d \mu\left(u_{1}\right)\right\} \\
= & \text { const. }\|h\|_{\infty}^{k}|\varphi(z)|^{-(n+1+m) / 2} \\
& \cdot\left\{J_{-(n+1-m) / 2,(n-2+m) / 2}(w)+|\log | \varphi(w) \| J_{-(n+1-m) / 2,(n+m) / 2}(w)\right\} \\
\leq & \text { const. }\|h\|_{\infty}^{k}|\varphi(z)|^{-(n+1+m) / 2} \\
& \cdot\left\{|\varphi(w)|^{-(n-2+m) / 2}+|\log | \varphi(w) \| \cdot|\varphi(w)|^{-(n+m) / 2}\right\}
\end{aligned}
$$

i.e.

$$
\left|I_{3}(z, w)\right| \leq \text { const. }\|h\|_{\infty}^{k}|\varphi(z)|^{-(n+1+m) / 2}|\varphi(w)|^{-(n+1+m) / 2} F(w) .
$$

Finally (as $H_{2}=H_{4}$ and $G_{3}=G_{4}$ )

$$
\left|I_{4}(z, w)\right| \leq \text { const. }\|h\|_{\infty}^{k}|\varphi(z)|^{-(n+1+m) / 2}|\varphi(w)|^{-(n+1+m) / 2} F(z) F(w) .
$$


The estimates (12)-(15) lead to

$$
\begin{aligned}
\left|\left[K_{1,|\varphi|^{m}}^{(k)} h^{(k)}\right](z, w)\right| \leq & \text { const. }\|h\|_{\infty}^{k}|\varphi(z)|^{-(n+1+m) / 2} \\
& \cdot|\varphi(w)|^{-(n+1+m) / 2}(1+F(z)+F(w)+F(z) F(w)) .
\end{aligned}
$$

To deal with $K_{1,|\varphi|^{m}}^{(1)}$ we firstly note that

$$
\left[K_{1,|\varphi|^{m}}^{(1)} h^{(1)}\right](z, w)=c_{\Omega}^{2} J_{1}(z, w)+c_{\Omega}\left(J_{2}(z, w)+J_{3}(z, w)\right)+J_{4}(z, w)
$$

where

$$
\begin{aligned}
J_{1}(z, w)= & \int_{\Omega}\left|\nabla \varphi\left(u_{1}\right)\right|^{4}\left|\operatorname{det} L_{\varphi}\left(u_{1}\right)\right|^{2} \Psi\left(z, u_{1}\right)^{-(n+1+m)} \\
& \cdot \overline{\Psi\left(w, u_{1}\right)}-(n+1+m) h\left(u_{1}\right)\left|\varphi\left(u_{1}\right)\right|^{m} d \mu\left(u_{1}\right) \\
J_{2}(z, w)= & \int_{\Omega}\left|\nabla \varphi\left(u_{1}\right)\right|^{2} \cdot \overline{\operatorname{det} L_{\varphi}\left(u_{1}\right)} \cdot E\left(z, u_{1}\right) \\
& \cdot \overline{\Psi\left(w, u_{1}\right)}-(n+1+m) h\left(u_{1}\right)\left|\varphi\left(u_{1}\right)\right|^{m} d \mu\left(u_{1}\right) \\
J_{3}(z, w)= & \int_{\Omega}\left|\nabla \varphi\left(u_{1}\right)\right|^{2} \cdot \operatorname{det} L_{\varphi}\left(u_{1}\right) \cdot \Psi\left(z, u_{1}\right)^{-(n+1+m)} \\
& \cdot \overline{E\left(w, u_{1}\right)} h\left(u_{1}\right)\left|\varphi\left(u_{1}\right)\right|^{m} d \mu\left(u_{1}\right) \\
J_{4}(z, w)= & \int_{\Omega} E\left(z, u_{1}\right) \overline{E\left(w, u_{1}\right)} h\left(u_{1}\right)\left|\varphi\left(u_{1}\right)\right|^{m} d \mu\left(u_{1}\right) .
\end{aligned}
$$

Then

$$
\begin{aligned}
& \left|J_{1}(z, w)\right| \\
& \leq \text { const. }\|h\|_{\infty} \int_{\Omega}\left|\Psi\left(z, u_{1}\right)\right|^{-(n+1+m)}\left|\Psi\left(w, u_{1}\right)\right|^{-(n+1+m)}\left|\varphi\left(u_{1}\right)\right| d \mu\left(u_{1}\right) \\
& \leq \text { const. }\|h\|_{\infty} \cdot\left(\int_{\Omega}\left|\Psi\left(z, u_{1}\right)\right|^{-2(n+1+m)}\left|\varphi\left(u_{1}\right)\right|^{m} d \mu\left(u_{1}\right)\right)^{1 / 2} \\
& \quad \cdot\left(\int_{\Omega}\left|\Psi\left(w, u_{1}\right)\right|^{-2(n+1+m)}\left|\varphi\left(u_{1}\right)\right|^{m} d \mu\left(u_{1}\right)\right)^{1 / 2} \\
& =\text { const. }\|h\|_{\infty} J_{m, n+1+m}(z)^{1 / 2} J_{m, n+1+m}(w)^{1 / 2}
\end{aligned}
$$

i.e.

$$
\left|J_{1}(z, w)\right| \leq \text { const. }\|h\|_{\infty}|\varphi(z)|^{-(n+1+m) / 2}|\varphi(w)|^{-(n+1+m) / 2} .
$$

Next 


$$
\begin{aligned}
&\left|J_{2}(z, w)\right| \\
& \leq \text { const. }\|h\|_{\infty} \int_{\Omega}\left|E\left(z, u_{1}\right)\right| \cdot\left|\Psi\left(w, u_{1}\right)\right|^{-(n+1+m)}\left|\varphi\left(u_{1}\right)\right|^{m} d \mu\left(u_{1}\right) \\
& \leq \text { const. }\|h\|_{\infty}\left(\int_{\Omega}\left|E\left(z, u_{1}\right)\right|^{2}\left|\varphi\left(u_{1}\right)\right|^{m} d \mu\left(u_{1}\right)\right)^{1 / 2} \\
& \cdot\left(\int_{\Omega}\left|\Psi\left(w, u_{1}\right)\right|^{-2(n+1+m)}\left|\varphi\left(u_{1}\right)\right|^{m} d \mu\left(u_{1}\right)\right)^{1 / 2} \\
&=\text { const. }\|h\|_{\infty} \cdot\|E(z, \cdot)\|_{m} J_{m, n+1+m}(w)^{1 / 2} \\
& \leq \text { const. }\|h\|_{\infty} \cdot\|E(z, \cdot)\|_{m}|\varphi(w)|^{-(n+1+m) / 2}
\end{aligned}
$$

On the other hand

$$
\begin{aligned}
\mid E(z, \cdot) \|_{m}^{2} & \leq \text { const. } \int_{\Omega}\left|\Psi\left(z, u_{1}\right)\right|^{-2(n+1+m)+1} \cdot|\log | \Psi\left(z, u_{1}\right) \|^{2}\left|\varphi\left(u_{1}\right)\right|^{m} d \mu\left(u_{1}\right) \\
\leq & \text { const. }\left(\int_{\Omega}\left|\Psi\left(z, u_{1}\right)\right|^{-2(n+1+m)+3}\left|\varphi\left(u_{1}\right)\right|^{m} d \mu\left(u_{1}\right)\right. \\
& \left.+|\log | \varphi(z) \|^{2} \int_{\Omega}\left|\Psi\left(z, u_{1}\right)\right|^{-2(n+1+m)+1}\left|\varphi\left(u_{1}\right)\right|^{m} d \mu\left(u_{1}\right)\right) \\
= & \text { const. }\left(J_{m, n-2+m}(z)+|\log | \varphi(z) \|^{2} J_{n, n+m}(z)\right) \\
\leq & \text { const. }|\varphi(z)|^{-(n+m)}\left(|\varphi(z)|^{2}+|\log | \varphi(z) \|^{2}\right)
\end{aligned}
$$

i.e.

$$
\|E(z, \cdot)\|_{m} \leq|\varphi(z)|^{-(n+1+m) / 2} F(z)
$$

We conclude that

$$
\left|J_{2}(z, w)\right| \leq \text { const. }\|h\|_{\infty}|\varphi(z)|^{-(n+1+m) / 2}|\varphi(w)|^{-(n+1+m) / 2} F(z) .
$$

Similarly

$$
\left|J_{3}(z, w)\right| \leq \text { const. }\|h\|_{\infty}|\varphi(z)|^{-(n+1+m) / 2}|\varphi(w)|^{-(n+1+m) / 2} F(w) .
$$

Finally

i.e.

$$
\left|J_{4}(z, w)\right| \leq \text { const. }\|h\|_{\infty}\|E(z, \cdot)\|_{m} \cdot\|E(w, \cdot)\|_{m}
$$

(20) $\quad\left|J_{4}(z, w)\right| \leq$ const. $\|h\|_{\infty}|\varphi(z)|^{-(n+1+m) / 2}|\varphi(w)|^{-(n+1+m) / 2} F(z) F(w)$.

By taking into account the estimates (17)-(20) it follows that (16) holds good for 
$k=1$ as well. To deal with $K_{1,|\varphi|^{m}}^{(2)} h^{(2)}$ one firstly uses the Schwarz inequality and Lemma 2.8 in [18], p. 233, so that to obtain

$$
\begin{aligned}
& \left|\left[K_{1,|\varphi|^{m}}^{(2)} h^{(2)}\right](z, w)\right| \\
& \quad \leq \text { const. }\|h\|_{\infty}^{2}|\varphi(z)|^{-(n+1+m) / 2} \int_{\Omega}\left|K_{m}\left(u_{1}, w\right)\right| \cdot\left|\varphi\left(u_{1}\right)\right|^{-(n+1-m) / 2} d \mu\left(u_{1}\right) .
\end{aligned}
$$

On the other hand

$$
\begin{aligned}
\int_{\Omega} \mid K_{m} & \left.\left(u_{1}, w\right)|\cdot| \varphi\left(u_{1}\right)\right|^{-(n+1-m) / 2} d \mu\left(u_{1}\right) \\
\leq & \text { const. }\left(\int_{\Omega}\left|\Psi\left(w, u_{1}\right)\right|^{-(n+1+m)}\left|\varphi\left(u_{1}\right)\right|^{-(n+1-m) / 2} d \mu\left(u_{1}\right)\right. \\
& \left.+\int_{\Omega}\left|\Psi\left(w, u_{1}\right)\right|^{-(n+1+m)+1 / 2}|\log | \Psi\left(w, u_{1}\right) \| \cdot\left|\varphi\left(u_{1}\right)\right|^{-(n+1-m) / 2} d \mu\left(u_{1}\right)\right) \\
= & \operatorname{const.}\left(J_{-(n+1-m) / 2,(n+1+m) / 2}(w)+J_{-(n+1-m) / 2,(n-2+m) / 2}(w)\right. \\
& \left.+|\log | \varphi(w) \| J_{-(n+1-m) / 2,(n+m) / 2}(w)\right) \\
\leq & \operatorname{const.}\left(|\varphi(w)|^{-(n+1+m) / 2}+|\varphi(w)|^{-(n-2+m) / 2}\right. \\
& \left.+|\log | \varphi(w) \| \cdot|\varphi(w)|^{-(n+m) / 2}\right)=\text { const. }|\varphi(w)|^{-(n+1+m) / 2}(1+F(w))
\end{aligned}
$$

hence we may conclude that (16) holds for $k=2$ as well. At this point (16) furnishes

$$
\begin{aligned}
& \sum_{k=1}^{\infty} \mid[\left.K_{1,|\varphi|^{m}}^{(k)} h^{(k)}\right](z, w) \mid \\
& \leq \text { const. } \frac{1}{1-\|h\|_{\infty}}|\varphi(z)|^{-(n+1+m) / 2}|\varphi(w)|^{-(n+1+m) / 2} \\
& \cdot(1+F(z)+F(w)+F(z) F(w))
\end{aligned}
$$

which, together with the estimate in Theorem 1, yields (10).

\section{The complex dilatation of a symplectomorphism and the Beltrami equations}

Let $\Omega \subset C^{n}$ be a $\gamma$-Kobayashi domain, for some $\gamma \in A W(\Omega)$. Let $F$ be a symplectomorphism of $\left(\Omega, \omega_{\gamma}\right)$ in itself. We have

LEMMA 3. For any $z \in \Omega$ and any $Z \in T^{1,0}(\Omega)_{z}, \quad Z \neq 0$, one has $\left(d_{z} F\right) \bar{Z} \notin T^{1,0}(\Omega)_{F(z)}$. 
The proof is by contradiction. Assume that $\left(d_{z} F\right) \bar{Z} \in T^{1,0}(\Omega)_{F(z)}$ for some $Z \in$ $T^{1,0}(\Omega)_{z}, \quad Z \neq 0$, and some $z \in \Omega$. As $F$ is a diffeomorphism $\left(d_{z} F\right) \bar{Z} \neq 0$. Hence

$$
\begin{aligned}
0 & <\left\|\left(d_{z} F\right) \bar{Z}\right\|^{2}=g_{\gamma, F(z)}\left(\left(d_{z} F\right) \bar{Z},\left(d_{z} F\right) Z\right) \\
& =-i \omega_{\gamma, z}(\bar{Z}, Z)=-\|Z\|^{2},
\end{aligned}
$$

a contradiction.

Let $T^{1,0}(\Omega)_{F}$ consist of all $Z \in T(\Omega) \otimes C$ with $(d F) Z \in T^{1,0}(\Omega)$.

Lemma 4. For any symplectomorphism $F$ of $\left(\Omega, \omega_{\gamma}\right)$ there is a $\boldsymbol{C}$-antilinear bundle map $\operatorname{dil}(F): T^{1,0}(\Omega) \rightarrow T^{1,0}(\Omega)$ so that

$$
T^{1,0}(\Omega)_{F}=\left\{Z-\overline{\operatorname{dil}(F) Z}: Z \in T^{1,0}(\Omega)\right\}
$$

To prove Lemma 4 , let $\pi_{0,1}: T(\Omega) \otimes C \rightarrow T^{0,1}(\Omega)$ be the natural projection. Then

$$
T^{1,0}(\Omega)_{F}=\operatorname{Ker}\left(\pi_{0,1} \circ(d F)\right) .
$$

Let $\left(z^{1}, \ldots, z^{n}\right)$ be the natural complex coordinates on $C^{n}$. Set

$$
F_{k}^{J}=\frac{\partial F^{J}}{\partial z^{k}}, \quad F_{\bar{k}}^{J}=\frac{\partial F^{J}}{\partial \bar{z}^{k}}
$$

etc.. Then $\operatorname{det}\left(F_{\bar{j}}^{\bar{k}}\right) \neq 0$ everywhere on $\Omega$. Indeed, if $\operatorname{det}\left(F_{\bar{j}}^{\bar{k}}\left(z_{0}\right)\right)=0$ at some $z_{0}$ $\in \Omega$ then $\sum_{k} F_{\bar{k}}^{j}\left(z_{0}\right) \overline{\zeta^{k}}=0,1 \leq j \leq n$, for some $\left(\zeta^{1}, \ldots, \zeta^{n}\right) \in C^{n}-\{0\}$. Set $Z=$ $\sum_{J} \zeta_{j}\left(\partial / \partial z^{J}\right)_{z_{0}} \in T^{1,0}(\Omega)_{z_{0}}$. Then $Z \neq 0$ and

$$
\left(d_{z_{0}} F\right) \bar{Z}=\sum_{j, k} \overline{\zeta^{k}} F_{\bar{k}}^{J}\left(z_{0}\right)\left(\frac{\partial}{\partial z^{J}}\right)_{F\left(z_{0}\right)} \in T^{1,0}(\Omega)_{F\left(z_{0}\right)},
$$

a contradiction (by Lemma 3). Let $\operatorname{dil}(F): T^{1,0}(\Omega) \rightarrow T^{1,0}(\Omega)$ be given by $\operatorname{dil}(F)\left(\partial / \partial z^{J}\right)=\sum_{k} \operatorname{dil}(F)_{j}^{k} \partial / \partial z^{k}$ (followed by $C$-antilinear extension) where

$$
F_{\bar{J}}^{\ell}=\sum_{k} \operatorname{dil}(F)_{J}^{k} F_{k}^{\ell} .
$$

Finally, note that $\partial / \partial z^{J}-\overline{\operatorname{dil}(F) \partial / \partial z^{J}} \in \operatorname{Ker}\left(\pi_{0,1} \circ(d F)\right)$.

q.e.d.

The bundle map $\operatorname{dil}(F)$ is referred to as the complex dilatation (of the symplectomorphism $F$ ).

Proposition 1. Let $F$ be a symplectomorphism of $\left(\Omega, \omega_{\gamma}\right)$ and $\operatorname{dil}(F)$ its complex dilatation. Then

$$
\omega_{\gamma}(Z, \overline{\operatorname{dil}(F) W})+\omega_{\gamma}(\overline{\operatorname{dil}(F) Z}, W)=0
$$

for any $Z, W \in T^{1,0}(\Omega)$. Also, $\operatorname{dil}(F)=0$ if and only if $F$ is holomorphic. 
Indeed, if $Z \in T^{1,0}(\Omega)$ then $(d F)(Z-\overline{\operatorname{dil}(F) Z}) \in T^{1,0}(\Omega)$. Therefore, as $\omega_{\gamma}$ vanishes on complex vector of the same type,

$$
\begin{aligned}
0 & =\omega_{\gamma}((d F)(Z-\overline{\operatorname{dil}(F) Z}),(d F)(W-\overline{\operatorname{dil}(F) W})) \\
& =\omega_{\gamma}(Z-\overline{\operatorname{dil}(F) Z}, W-\overline{\operatorname{dil}(F) W})=-\omega_{\gamma}(Z, \overline{\operatorname{dil}(F) W})-\omega_{\gamma}(\overline{\operatorname{dil}(F) Z}, W)
\end{aligned}
$$

for any $Z, W \in T^{1,0}(\Omega)$.

By (21), each component $F^{J}$ of the symplectomorphism $F$ satisfies the first order PDE (with variable coefficients)

$$
\frac{\partial f}{\partial \bar{z}^{j}}=\sum_{k} d_{j}^{k} \frac{\partial f}{\partial z^{k}}
$$

where $d_{\bar{j}}^{k}=\operatorname{dil}(F)_{j}^{k}$. We refer to (22) as the Beltrami equations (cf. e.g. [20]). On the other hand, with any contact transformation $F: M \rightarrow N$ between two strictly pseudoconvex CR manifolds $M$ and $N$ one may associate (cf. [10], p. 61) a complex dilatation $\mu: T_{1,0}(M) \rightarrow T_{1,0}(M)$ and whenever $M=\boldsymbol{H}_{n-1}$ (the Heisenberg group) and $N$ is a real hypersurface in $C^{n}$ (carrying the standard CR structure induced from the complex structure of $C^{n}$ ), the components $F^{J}$ of $F$ satisfy the PDE

$$
L_{\bar{\alpha}} f=\sum_{\beta=1}^{n-1} \mu_{\bar{\alpha}}^{\beta} L_{\beta} f
$$

where $L_{\bar{\alpha}}=\partial / \partial \bar{z}^{\alpha}-i z^{\alpha} \partial / \partial t$ are the Lewy operators (cf. e.g. [5], p. 435-436) on $\boldsymbol{H}_{n-1}$, and $\mu L_{\alpha}=\sum_{\beta} \mu_{\bar{\alpha}}^{\beta} L_{\beta}$. We refer to (23) as the tangential Beltrami equations.

Consider the Siegel domain $\Omega_{n}=\left\{\varphi_{n}<0\right\}$ and let $F=\left(F^{1}, \ldots, F^{n}\right)$ be a symplectomorphism of $\left(\Omega_{n}, \omega_{\alpha}\right)$ in itself. Let $\mathscr{F}_{n}$ be the foliation of $\boldsymbol{C}^{n}$ by level sets of $\varphi_{n}$. If $F$ is smooth up to $\partial \Omega_{n}$ then $\mu$ (the complex dilatation of $F$ ) restricted to $T_{1,0}\left(\mathscr{F}_{n}\right)$ converges to the complex dilatation of the boundary contact transformation (the proof is a word by word repetition of the proof of Proposition 2 in [11], p. 1122). Also, if $\phi: \boldsymbol{H}_{n-1} \rightarrow \partial \Omega_{n}$ is the CR isomorphism $\phi(z, t)=\left(t+i|z|^{2}, z\right)$, then each $F^{\jmath} \circ \phi$ satisfies the tangential Beltrami equations (23) (this follows from the remark at the end of section 2 and by a result in [10], p. 62).

Let $d_{\bar{j}}^{k}$ be smooth functions defined on some neighborhood of $\bar{\Omega}_{n}$. The complex vector fields $\partial / \partial \bar{\zeta}^{j}-\sum_{k} d_{j}^{k} \partial / \partial \zeta^{k}$ span a rank $n$ complex vector subbundle $B \subset T\left(\Omega_{n}\right) \otimes C$. For the Siegel domain $\Omega_{n}$, the vector field $\xi$ (determined by $(6))$ is given by $\xi=2 i \partial / \partial \zeta^{1}$. The CR isomorphism $\phi: \boldsymbol{H}_{n-1} \approx \partial \Omega_{n}$ maps the Lewy operators $L_{\bar{\alpha}}$ into $Z_{\bar{\alpha}}=\partial / \partial \bar{\zeta}^{\alpha}+\zeta^{\alpha} \bar{\xi}, 2 \leq \alpha \leq n$. We establish the following

Proposition 2. Let $D$ be an open neighborhood of $\bar{\Omega}_{n}$ and $\mu: T^{1,0}(D) \rightarrow$ $T^{1,0}(D)$ a fibrewise $C$-antilinear bundle morphism which maps $T_{1,0}\left(\partial \Omega_{n}\right)$ into itself. Let $B_{b} \subset T\left(\partial \Omega_{n}\right) \otimes C$ be the rank $n-1$ complex subbundle spanned 
by $Z_{\bar{\alpha}}-\mu_{\bar{\alpha}}^{\beta} Z_{\beta}, \quad 2 \leq \alpha \leq n$, where $\mu_{\bar{\alpha}}^{\beta}$ are given by $\mu\left(Z_{\alpha}\right)=\mu_{\bar{\alpha}}^{\beta} Z_{\beta}$. Let $d_{\bar{j}}^{k}$ be given by $\mu\left(\partial / \partial \zeta^{J}\right)=d_{\bar{j}}^{k} \partial / \partial \zeta^{k}$ and set $h(\zeta)=2 i \sum_{\beta} d_{\overline{1}}^{\beta} \bar{\zeta}_{\beta}-d_{\overline{1}}^{1}-1$. Then

$$
B_{b}=\left[T\left(\partial \Omega_{n}\right) \otimes C\right] \cap B
$$

on $\partial \Omega_{n} \cap\{\zeta: h(\zeta) \neq 0\}$. In particular, the trace on $\partial \Omega_{n}$ of any solution $f \in$ $C^{\infty}\left(\bar{\Omega}_{n}\right)$ of the Beltrami equations (22) satisfies the tangential Beltrami equations $Z_{\bar{\alpha}} f=\mu_{\bar{\alpha}}^{\beta} Z_{\beta} f$ on the open set $\left\{\zeta \in \partial \Omega_{n}: h(\zeta) \neq 0\right\}$.

Indeed, as $\mu\left(T_{1,0}\left(\partial \Omega_{n}\right)\right) \subseteq T_{1,0}\left(\partial \Omega_{n}\right)$,

$$
\begin{aligned}
\mu_{\bar{\alpha}}^{\beta} & =d_{\bar{\alpha}}^{\beta}-2 i \zeta_{\alpha} d_{\overline{1}}^{\beta}, \\
2 i \mu_{\bar{\alpha}}^{\beta} \bar{\zeta}_{\beta} & =d_{\bar{\alpha}}^{1}-2 i \zeta_{\alpha} d_{\overline{1}}^{1},
\end{aligned}
$$

where $\zeta_{\alpha}=\zeta^{\alpha}$. Consequently $Z=a^{J}\left(\partial / \partial \bar{\zeta}^{J}-d_{j}^{k} \partial / \partial \zeta^{k}\right)$ is tangent to $\partial \Omega_{n} \cap$ $\{h \neq 0\}$ if and only if $a^{1}=-2 i \zeta_{\alpha} a^{\alpha}$, i.e. $Z \in \Gamma^{\infty}\left(B_{b}\right) . \quad$ q.e.d.

Proposition 3. Let $F: \Omega_{n} \rightarrow \Omega_{n}$ be a $C^{\infty}$ diffeomorphism, smooth up to the boundary, each of whose components $F^{J}$ satisfies the PDE

$$
Z_{\bar{\alpha}} F^{J}=\mu_{\bar{\alpha}}^{\beta} Z_{\beta} F^{J}
$$

in $\Omega_{n}$, for some $C^{\infty}$ functions $\mu_{\bar{\alpha}}^{\beta}: \Omega_{n} \rightarrow C$. If $F$ is a foliated map, i.e. it preserves the foliation $\mathscr{F}_{n}$, then for any $\alpha>-1$ there is $f_{\alpha} \in C^{2}\left(\Omega_{n}\right), f_{\alpha} \neq 0$ everywhere, so that

$$
F^{*} \omega_{\alpha} \equiv f_{\alpha} \omega_{\alpha}, \bmod \theta_{\alpha}, d \rho_{\alpha}
$$

Proof. Set $V_{\alpha}=Z_{\alpha}\left(F^{J}\right) \partial / \partial \zeta^{J}$ and $W_{\alpha}=Z_{\bar{\alpha}}\left(F^{J}\right) \partial / \partial \zeta^{J}$. As

$$
\begin{gathered}
(d F) T\left(\mathscr{F}_{n}\right)=T\left(\mathscr{F}_{n}\right), \\
Z_{\alpha}-\mu_{\alpha}^{\bar{\beta}} Z_{\bar{\beta}} \in T\left(\mathscr{F}_{n}\right) \otimes C, \\
W_{\alpha}=\mu_{\bar{\alpha}}^{\beta} V_{\beta},
\end{gathered}
$$

(where $\mu_{\alpha}^{\bar{\beta}}=\overline{\mu_{\bar{\alpha}}^{\beta}}$ ) one has

$$
(d F)\left(Z_{\alpha}-\mu_{\alpha}^{\bar{\beta}} Z_{\bar{\beta}}\right)=V_{\alpha}-\mu_{\alpha}^{\bar{\beta}} W_{\beta} \in T_{1,0}\left(\mathscr{F}_{n}\right) .
$$

Note that

$$
H\left(\mathscr{F}_{n}\right) \otimes C=\operatorname{Re}\left\{B_{b} \oplus \overline{B_{b}}\right\}
$$

and

$$
\left(F^{*} \theta_{\alpha}\right) \bar{B}_{b} \subseteq \theta_{\alpha}\left(T_{1,0}\left(\mathscr{F}_{n}\right)\right)=0
$$

hence

$$
F^{*} \theta_{\alpha}=a \theta_{\alpha}+b d \rho_{\alpha}
$$


for some $C^{\infty}$ functions $a, b: \Omega_{n} \rightarrow \boldsymbol{R}$. Here $\theta_{\alpha}=(i / 2)(\bar{\partial}-\partial) \rho_{\alpha}$. Also $\rho_{\alpha} \circ F=\lambda \rho_{\alpha}$ for some $\lambda \in C^{2}\left(\bar{\Omega}_{n}\right), \lambda>0$ everywhere. Next, one may use

to conclude that

$$
\omega_{\alpha}=(n+1+\alpha)\left\{\frac{d \theta_{\alpha}}{\rho_{\alpha}}-\frac{d \rho_{\alpha} \wedge \theta_{\alpha}}{\rho_{\alpha}^{2}}\right\}
$$

$$
F^{*} \omega_{\alpha}=\frac{a}{\lambda} \omega_{\alpha}+\frac{n+1+\alpha}{\lambda \rho_{\alpha}}\left((d a-a d \log \lambda) \wedge \theta_{\alpha}+(d b-b d \log \lambda) \wedge d \rho_{\alpha}\right) .
$$

Finally $a \neq 0$ everywhere (for if $a\left(z_{0}\right)=0$ at some $z_{0} \in \Omega_{n}$ then

$$
\theta_{\alpha, F\left(z_{0}\right)}\left(d_{z_{0}} F\right)=b\left(z_{0}\right) d_{z_{0}} \rho_{\alpha},
$$

i.e. $\left(d_{z_{0}} F\right) T\left(\mathscr{F}_{n}\right)_{z_{0}} \subseteq H\left(\mathscr{F}_{n}\right)_{F\left(z_{0}\right)}$, a contradiction).

\section{REFERENCES}

[1] E. Barletta and S. Dragomir, On the Djrbashian kernel of a Siegel domain, Studia Math., 127 (1998), 47-63.

[2] S. Bergman, The Kernel Function and Conformal Mapping, Math. Surveys, 5, Amer. Math. Soc., New York, 1950.

[ 3 ] S. Dragomir, On weighted Bergman kernels of bounded domains, Studia Math., 107 (1994), 149-157

[4] C. Feferman, The Bergman kernel and biholomorphic mappings of pseudoconvex domains, Invent. Math., 26 (1974), 1-65.

[ 5 ] G. B. Folland AND E. M. STEIN, Estımates for the $\bar{\partial}_{b}$-complex and analysis on the Heisenberg group, Comm. Pure Appl. Math., 27 (1974), 429-522.

[6] F Forelli AND W Rudin, Projections on spaces of holomorphic functions in balls, Indiana Univ. Math. J., 24 (1974), 593-602.

[7] S. Helgason, Differential geometry, Lie Groups, and Symmetric Spaces, Academic Press, New York, 1978, 352-373.

[ 8 ] S. Kobayashi, Geometry of bounded domains, Trans. Amer. Math. Soc., 92 (1959), 267-290.

[9] A. KorÁNYI AND H. M. REIMANN, Quasiconformal mappings on the Heisenberg group, Invent. Math., 80 (1985), 309-338.

[10] A. KorÁnYI AND H. M. REIMANN, Quasiconformal mappıngs on CR manifolds, Lecture Notes in Math., 1422, Sprınger-Verlag, Berlin-Heidelberg-New York, 1988, 59-75.

[11] A. Korányi and H. M. ReimanN, Contact transformations as limits of symplectomorphisms, C.R. Acad. Sc1. Parıs, 318 (1994), 1119-1124.

[12] A. KorÁnYi AND H. M. ReimanN, Foundations for the theory of quasiconformal mappings on the Heisenberg group, Adv. Math., 111 (1995), 1-87

[13] J. M. LeE and R. Melrose, Boundary behaviour of the complex Monge-Ampère equation, Acta Math., 148 (1982), 159-192.

[14] E. LigocKA, On the Forelli-Rudin construction and weighted Bergman projections, Studia Math., 94 (1989), 257-272.

[15] T. Mazur, On complex manifolds of Bergman type, Classical Analysis, World Scientific, River Edge, 1992, 132-138.

[16] Z. PASTERnaK-Winiarski, On the dependence of the reproducing kernel on the weight of integration, J. Funct. Anal., 94 (1990), 110-134. 
[17] Z. Pasternak-Winiarski, On weights which admit the reproducing kernel of Bergman type, Internat. J. Math. Math. Sc1., 15 (1992), 1-14.

[18] M. M. Peloso, Hankel operators on weighted Bergman spaces on strongly pseudoconvex domains, Illino1s J. Math., 38 (1994), 223-249.

[19] M. M. Peloso, Sobolev regularity of the weighted Bergman projections and estımates for minımal solutions to the $\vec{\partial}$-equation, Complex Variables Theory Appl., 27 (1995), 339-363.

[20] L. WANG, The globally homeomorphic solutions to Beltramı system in $C^{n}$, Math. Z., 216 (1994), 371-377

UNiVERSità degli STUdi della Basilicata

Dipartimento di MATEMATICA

VIA N. SAuro 85

85100 PotenZA

ITALY

e-mail: barletta@unibas.1t

e-mail: dragomır@unibas.1t 